\title{
Research 'essential' for control strategy
}

Two main research goals are most likely to lead in the long term to large strides in malaria control. One is a vaccine, and the second is likely to be the genetic engineering of mosquitoes to confer resistance to the parasite. And there is growing awareness that a more science-based approach is needed in general if malaria control techniques are to be implemented effectively.

One glaring need is better understanding of the complex interaction between malaria transmission rates, acquired immunity, and the clinical forms and incidence of the disease. In regions such as sub-Saharan Africa, where an individual may received up to 1,000 infectious bites a year, many people slowly acquire a natural immunity that allows them to tolerate a parasite load without developing severe symptoms.

When such populations experience prolonged periods of low transmission, individuals can quickly lose their immunity and risk developing severe malaria if transmission rates increase again. This fact has enormous implications for both the success of control measures, and the understanding of the frequent epidemics triggered by fluctuations in climate.

Some scientists argue that control methods such as insecticide-impregnated bed-nets - which have been shown to cut mortality and morbidity by 15 to 33 per cent in short-term trials - may in the long term only make populations more vulnerable to severe disease by reducing natural immunity. If they are correct, methods such as bed-nets and genetically engineered mosquitoes would have little impact on the control of the disease, and indeed could even make matters worse.

This complex debate seems to be going the way of those who feel that the lives saved immediately justifies taking the risk. But it has revealed the overriding need to accompany vector control programmes with rigorous long-term epidemiological studies of their impact on immunity, morbidity and mortality.

The same need applies to urban malaria, where transmission seems to be reduced, but severe and fatal disease more frequent. This may have important public health significance, given that migration from rural to urban areas is expected to be the biggest social change in Africa in the coming decades.

The need to understand the complex dynamics of malaria is also acute in other sectors. There is growing awareness that proper testing of candidate vaccines, and successful introduction of any working vaccine, will require much more research on epidemiology, parasite polymorphism, and mechanisms of immunity and transmission, and knowledge of how these vary from region to region.

More broadly, there is growing awareness that control needs to be more science-based. Richard Feachem of the World Bank says that a comprehensive and integrated approach is needed, not what he describes as "a thoughtless grabbing of the latest attractive idea and just putting it into the field without adequate care".

Harold Varmus, director of the US National Institutes of Health, describes moves to develop control programmes without a strong emphasis on research as "penny wise and pound foolish. I don't think you can do malaria control without a research component, because we don't really know the best way to do control."

Indeed, many believe that what is now needed is an integrated approach, stretching from better diagnosis and treatment of sick children in the home to rational drug use to limit the spread of resistance, monitoring and evaluating control measures, and developing tools such as drugs and vaccines through basic research. The simplistic notion "that a magic bullet developed in a laboratory in London or Washington would bring an end to malaria is now obsolete", says Luiz Pereira da Silva, a vaccine researcher at the Institut Pasteur in Paris.

\section{Vaccines: a roller-coaster of hopes}

After many years of frustration, malaria researchers are excited at the prospects raised by a preliminary evaluation of a vaccine developed by SmithKline Beecham Biologicals and the US Army, which protected six out of seven volunteers subjected to repeated bites by infected mosquitoes.

The vaccine is based on the the major surface protein of sporozoites, the circumsporozoite protein, which helps them infect hepatocytes. Previous vaccines using this protein had failed in trials because they did not elicit a sufficient immune response. But the new study suggests that this obstacle might be overcome using adjuvants, compounds which stimulate the immune system. "It's a tremendously exciting development", says Ripley Ballou, head of immunology at Walter Reed.

The study tested not just the vaccine but also three different adjuvants. Three groups of subjects were assigned to groups differing only in the adjuvant used. The six out of seven protected were all given a proprietary adjuvant codenamed QS21 (all six control volunteers became infected). This underlines the important role that adjuvants have in protection, not just in vaccines for malaria, but for many other diseases.

\section{IMAGE UNAVAILABLE FOR COPYRIGHT REASONS}

Current trials will be watched carefully for signs of progress in the hunt for a vaccine.

The early quest for a vaccine built on the observation that people repeatedly bitten by infectious mosquitoes gradually acquire immunity over a period of many years. Moreover, in the 1960s the British scientists Sydney Cohen and Sir Ian Mc Gregor discovered that antibodies from such individuals could reduce the parasite load in people lacking immunity and clear disease symptoms.

Around the same time, researchers at New York University (NYU) achieved full protection for the first time, using animal models - by injecting small numbers of sporozoites derived from mosquitoes that had previously been irradiated. Later, researchers at the University of Maryland, NYU and the Walter Reed Army Institute showed that 90 per cent of a group of human volunteers challenged with sporozoites from irradiated mosquitoes later resisted exposure to virulent sporozoites.

\section{Search for 'sporozoite' vaccines}

But this was hardly a practical field strategy; inducing resistance required exposing volunteers to several hundred bites by irradiated mosquitoes over several months. The more practical alternative of culturing millions of sporozoites, irradiating them directly, and formulating a vaccine, was also precluded, as sporozoites were impossible to culture (as they still are).

But the successes sent researchers scurrying to their laboratories to find sporozoite proteins that would reproduce the effect of whole 